\title{
Dispositivos móveis na escola: redefinição de ambientes e métodos de aprendizagem no contexto italiano*
}

\author{
Andrea Garavaglia**
}

\section{Resumo}

Este artigo discute alguns estudos recentes que permite compreender a importância da análise das necessidades educativas na concepção da sala de aula digital e suas possíveis redefiniçôes nos ambientes e métodos de aprendizagem. A reflexão refere-se à configuraçáo e implementaçáo de ambientes educativos inovadores por meio de tecnologias móveis na escola italiana. Recentemente, o foco no ensino ativo e na aprendizagem informal com a integração de dispositivos móveis tem-se refletido na metodologia da Flipped Lesson (aula invertida) e dos Episódios de Aprendizagem Situada (EAS), alguns dos métodos mais interessantes para apoiar a renovação da prática escolar. Estes métodos são baseados em trabalhos de grupo realizados em sala de aula e em casa com apoio de dispositivos digitais e em alguns casos, com o, Bring Your Own Device (BYOD) (Traga seu próprio dispositivo). A escolha do método de ensino adequado aos desafios atuais é fundamental em uma sociedade onde o uso da internet aumenta a cada dia e a possibilidade de estar sempre conectado propicia processos de aprendizagem difusa.

Palavras-chave: Escola. Dispositivos móveis. Aprendizagem.

\footnotetext{
* Texto traduzido por Monica Fantin, do Centro de Educação da Universidade Federal de Santa Catarina (UFSC).

** Professor daUniversità degli Studi di Milano-Bicocca, em Milão, Itália.
} 


\section{Introdução}

O âmbito de debate sobre a predisposição de ambientes didáticos inovadores por meio da tecnologia nos últimos evoluiu ao lado das diversas experiências em que as tecnologias "entraram" em sala de aula. Particularmente na Itália é possível lembrar as iniciativas do Istituto Nazionale di Documentazione, Innovazione e Ricerca ${ }^{1}$ (INDIRE) relacionadas à "Escola Digital" (Programas Lousa Interativa Multimídia, LIM, e Cl@ssi 2.0), de onde emergiram as primeiras problemáticas de uma cansativa e lenta transformação, juntamente com alguns resultados interessantes que confirmaram o papel central do professor e do valor agregado das tecnologias para as didáticas ativas e colaborativas no ensino (RIVOLTELLA et al., 2012).

Recentemente, a recuperação da didática ativa e das aprendizagens informais integradas ao uso dos dispositivos móveis podem ser vistas nas abordagens da Flipped Lesson ou Aulas invertidas ${ }^{2}$ (MAZUR, 1991) e dos Episódios de Aprendizagem Situada $(\mathrm{EAS})^{3}$ (RIVOLTELLA, 2013), que são alguns dos métodos mais interessantes para apoiar a renovação da prática escolar baseada no trabalho de grupo em sala de aula e no trabalho realizado em casa com os dispositivos digitais. A particularidade dessas abordagens consiste em inverter a metodologia tradicional, substituindo a explicaçáo expositiva em sala de aula pelo estudo individual em casa, e o tempo da sala de aula é organizado prioritariamente para administrar as tarefas de grupo com o uso dos dispositivos móveis já difundidos entre os jovens. Sendo assim, neste artigo analisa-se algumas mudanças que estão ligadas à presença e propagação da rede, à difusão de dispositivos móveis e à discussão dos modelos das "salas de aula digitais", bem como os problemas ligados aos seus processos de planejamento no contexto das escolas italianas.

\section{As principais mudanças: a difusão da rede}

O cenário atual é decisivamente diferente daquele do início dos anos 2000, quando a rede entrava nas casas, mas ainda não era "móvel", e, em alguns casos, era limitada no tempo e a banda larga disponível era inferior a 5\% das médias que temos hoje. Apesar dessa evolução, estamos diante de um estranho paradoxo, pois embora muitas salas de aula náo estejam conectadas à internet - diferente da maioria dos espaços de trabalho dos pais e de grande parte das casas dos alunos - os laboratórios de informática ainda são o lugar destinado às aulas em que se usam as tecnologias, em muitos casos, a situação é bastante heterogênea: existem contextos escolares em que se pode viver cotidianamente um processo didático transmídia (JENKINS, 2010; GARAVAGLIA, 2014), graças aos dispositivos e à conexão sempre disponível, em 
outros os recursos financeiros dos estudantes compensam os da escola (perspectiva do BYOD), e outros ainda em que não há conexão e, no máximo, deve-se contentar com alguma possibilidade interativa (lousa digital) ou com o computador em sala de aula.

A sala de aula-online prevista nas recentes propostas educativas do contexto italiano parece tornar ainda mais crítico o processo de superação da situação atual de exclusão-inclusão da rede, em que a exclusão caracteriza os contextos escolares "não conectados" e a inclusão em rede acontece frequentemente graças aos smartphones dos alunos.

Neste cenário, é possível identificar um segundo nível de exclusão digital. $\mathrm{Na}$ verdade os sistemas formativos que sempre podem dispor de conexão "always on" apresentam lógica de continuidade temporal muito mais fluida, que permite aos alunos trabalhar com os mesmos artefatos digitais na sala de aula e em casa, permitindo maior flexibilidade de escolhas didáticas e opçóes educativas.

\section{Sempre conectado, aprender sempre? A aprendizagem difusa}

O conceito de aprendizagem difusa, pervasive learning, foi proposto por Dan Pontefract (2013), e o autor argumenta que a aprendizagem é um processo contínuo que toma forma em três dimensôes, na especificidade formal, informal e social. De acordo com Pontefract (2013, p. 27), na verdade:

[...] não importa se está em sala de aula, ou no meio de outras pessoas, se está sozinho ou se está trabalhando, a tecnologia e a aprendizagem estão realmente se tornando inseparáveis. Os puristas podem não concordar com estes argumentos, e também muitas outras pessoas clamam para poder voltar aos "velhos tempos" quando a aprendizagem era transmitida em um artigo na cátedra [...]. Aqueles dias estão desaparecendo rapidamente. Certamente o establishment, as instituiçóes educativas e as organizaçôes de controle e comando clássico continuarão o seu trabalho brutal, e nós dificilmente conseguiremos reconhecer o que está realmente acontecendo.

A disseminação da aprendizagem é, portanto, a tomada de consciência do fato que vivemos em um processo transmidiático contínuo (GARAVAGLIA, 2014), em que se aprende principalmente de acordo com as nossas necessidades e de acordo com o nosso tempo de aprendizagem. O papel dos dispositivos móveis também é reconhecido como fundamental para manter a continuidade funcional das próprias necessidades. 
Em tal perspectiva, os espaços formais, como as salas de aula e os ambientes de aprendizagem em rede, são os lugares onde o conhecimento é efetuado e rediscutido; os ambientes informais, por sua vez, incluem tutoriais, podcasts, webinars e outros recursos que a rede oferece para aprofundar e personalizar a aprendizagem; e, finalmente, a nível social, graças aos blogs, wikis e social network, o sujeito pode discutir, debater e tornar-se prosumer criando e compartilhando novos conteúdos. O sistema é cíclico, e, portanto, o que é aprofundado, discutido e produzido a nível informal e social é retomado em âmbito formal.

O pensamento de Pontefract (2013) é lúcido, linear e coerente com muitas instâncias transmidiáticas, e as fases por ele descritas parecem criar os pressupostos para construir percursos basesados no método Flipped Lesson ou EAS. Parece também resolver de modo ainda mais convincente o dilema-formal informal que alguns autores como Cross (2006) haviam enfrentado, alterando o peso da balança no sentido de um entusiasmo excessivo e desapaixonado pela aprendizagem formal.

Figura 1 - A distribuiçáo dos diferentes ambientes online e offline nas fases do modelo Pervasive Learning/Aprendizagem Difusa

\section{Pervasive Learning}

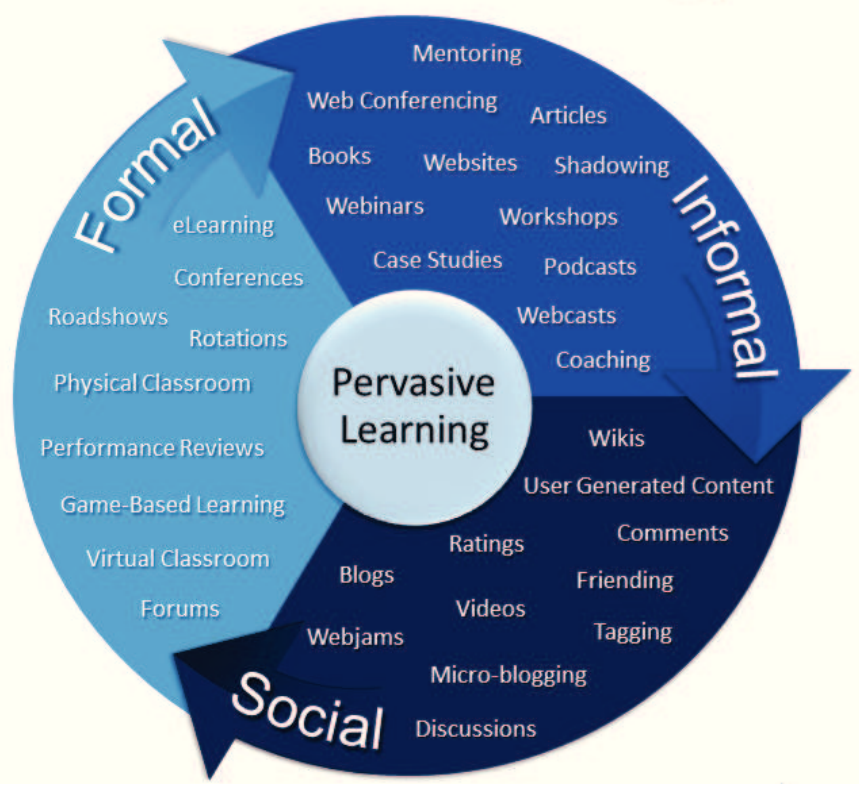

Fonte: Pontefract, 2013, p. 209. 
Na escola, o modelo de Pontefract (2013) parece aplicável, sobretudo, em nível secundário, quando os alunos adquirem autonomia e as competências mínimas para se administrarem na web.

\section{As principais mudanças: a propagação dos dispositivos móveis}

Este importante processo de integração das novas mídias em sala de aula é caracterizado pela difusão e propagação de smartphones e tablets entre os próprios alunos: os chamados dispositivos móveis, na prática, estão se tornando os dispositivos tecnológicos pessoais mais facilmente acessíveis e utilizáveis durante as aulas (KUKULSKA-HULME; TRAXLER, 2013). Na verdade, os dispositivos móveis estão produzindo o fenômeno da efemeridade no último decênio: com este termo o arquiteto e inventor Buckminster Fuller (1973) argumentava que o homem estava destinado a "fazer mais com menos", justamente o que está acontecendo com a miniaturização constante da tecnologia ao possibilitar a disseminação de mini-dispositivos pessoais capazes de desenvolver com eficácia tarefas cada vez mais complexas.

Embora os dispositivos móveis náo substituam o ambiente desktop pela completude e complexidade das funçóes, este potencial em crescimento é cada vez mais reconhecido pelas pessoas em todos os estratos sociais. Isso explica o aumento constante de acesso à internet via smartphones e tablets que, recentemente, obteve ainda resultado maior que o acesso via desktop, segundo o relatório de 2014, The digital consumer, de Nielsen ${ }^{4}$. Mesmo no âmbito profissional, em essência, está ocorrendo uma mudança de algumas atividades do desktop aos dispositivos móveis, e diferentes cursos de formação e capacitação destacam a possibilidade de usufruir atividades didáticas por meio de aplicativos para smartphones e tablets. Esta dinâmica implica a necessidade de construir experiências formativas integradas, com passagens significativas entre os diferentes ambientes, de modo a manter a coerência entre os diversos fluxos transmidiáticos.

Figura 2 - O infográfico evidencia a superaçáo das horas de consumo da rede por meio de um smartphone em relaçáo ao acesso pelo desktop: diferença de consumo expressa em horas no ano de 2014

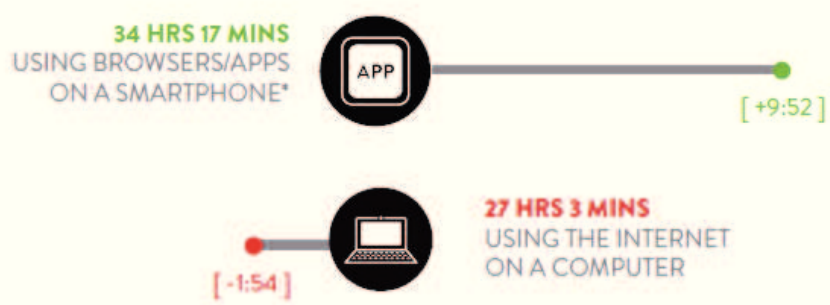

Fonte: The Nielsen Company, 2014, p. 9. 


\section{A sala de aula aberta: ponto de partida, retorno ou estímulo a novos modelos?}

$\mathrm{Na}$ última década, a atenção na configuração do ambiente das salas de aula cresceu juntamente com o desenvolvimento de modelos de "aula digital" (HYMAN, 2014; JOHN; WHEELER, 2008; MALCOM, 2010), colocando em discussão o padrão tradicional que foi estabelecido internacionalmente para trazer uma nova atenção ao conceito de salas de aula abertas.

A ideia de "Sala de aula Aberta" nasceu na Inglaterra, após a Segunda Guerra Mundial, a partir dos movimentos inovadores baseados na educação informal e se espalhou entre os anos 1960 e 1970, principalmente na América do Norte. É interessante observar que nos Estados Unidos da América, a construção de escolas com base neste conceito foi promovida na esteira do ativismo como resposta às aulas tradicionais (pautados no professor) que suprimiam a criatividade dos estudantes, e por isso era necessário estabelecer um ensino mais centrado no aluno (CUBAN, 2004).

Outro elemento interessante é o fato que na sala de aula aberta americana e inglesa náo foram adotados testes padronizados, nem currículos detalhados: os professores preferiam preparar a configuração de um ambiente sempre acessível que permitisse aos alunos entrar em contato com todos os objetos (livros, ferramentas), bem como áreas e ilhas chamadas "centros de interesse" utilizados para trabalhos específicos. Esses ambientes eram estruturados conforme as atividades propostas, tanto individualmente como em grupo. Diversas escolas foram construídas com a participação de professores juntamente com os arquitetos (seguindo as instâncias do design participativo) até 1970, quando devido à recessão econômica que aumentou a degradação social e os episódios de violência entre os jovens, a opinião americana sobre a utilidade de tal modelo educativo mudou. E, assim, no curso de uma década quase todas as escolas foram reconvertidas em tradicionais agregando as paredes que faltavam.

Recentemente, no contexto norte-europeu, verifica-se um renovado interesse nesse tipo de abordagem, graças ao desenvolvimento de uma escola de arquitetura mais centrada nas necessidades das pessoas e da sociedade local. Em alguns casos, a construção desse tipo de escola faz parte de uma resposta às necessidades dos cidadãos, como em Grundarfjörður, localizada na península islandesa de Snaefellsnes, onde a baixa densidade populacional e o clima difícil favorece a criaçáo de espaços comuns e flexíveis, usados durante todo o dia pelos habitantes do lugar. Seguramente, tal proposta é interessante para a ampla flexibilidade didática, pois as salas de aula são espaçosas e organizadas em mesas redondas, em ilhas de trabalho, e prateleiras da Pine Jog Elementary School, de Palm Beach, na Flórida, ou em salas multifuncionais, de Het 
4e Gymnasium, em Amsterdã (KRAMER, 2009). Em outros casos, no entanto, decidiu-se criar ambientes semelhantes àqueles espaços de trabalhos em multinacionais líderes da área de Tecnologia e Informação (TI) (como o Google): os mais famosos são aqueles projetados pelo arquiteto Rosan Bosch $\mathrm{Vittra}^{5}$, uma organização de escolas suecas que baseia os próprios ambientes na ideia de que as crianças devem poder expressar a sua própria criatividade e suas próprias ideias. Nessas escolas as tecnologias também são introduzidas massivamente, com a convicção que "tudo" pode contribuir para pensar nos processo de aprendizagem.

\section{Relação ambiente de sala de aula-método}

A experiência do Open Classroom, sala de aula aberta, permitiu refletir sobre a influência dos ambientes na qualidade do ensino: pensar que o processo formativo pode ser diretamente predeterminado de modo pontual pela estrutura arquitetônica é certamente discutível. O próprio Silberman (1973), autor de um cuidadoso estudo sobre a sala de aula aberta, destacou diversas vezes que, basicamente, a renovaçáo consistia em aderir a uma abordagem de ensino e aprendizagem. Portanto, as "escolas ou salas de aula abertas", podem não ser suficientes: se o professor não aderir a "esta abordagem" poderia sentir-se em desconfortável "prisão didática”. Um professor que constrói vínculos e supera as tensóes da sala de aula, de preferência discutindo as soluçôes possíveis com os alunos para fazer melhor cada trabalho, poderia construir um ambiente muito mais favorável e, paradoxalmente, "aberto", mesmo em condiçóes precárias (GARAVAGLIA, 2013).

\section{Configuração de ambientes flexíveis e ambientes rígidos: quais modelos para a sala de aula digital?}

A convergência de interesses para a renovação dos ambientes das salas de aula e a difusão de tecnologias educativas e móveis é um fenômeno essencialmente difundido a nível mundial. Sempre levando em conta as diferenças culturais, os diversos sistemas educativos e, naturalmente, as diferenças econômicas, fatores que podem influenciar notavelmente a configuração dos ambientes resultantes (BURKE; BURKE-SAMIDE, 2004).

Algumas configuraçôes de ambiente encontram melhor viabilidade, porque, por um lado são compatíveis com as exigências logísticas e organizativas das instituiçôes, e por outro porque são mais fáceis de tratar: entre estas, a configuração do 
one-to-one computing (PENUEL, 2006), que pode ser traduzida como o modelo 1:1, ou "um computador/artefato por aluno".

À luz deste discurso, é interessante aprofundar os modelos de configuração de ambientes possíveis com a tecnologia. Subjacente a essa sistematização (GARAVAGLIA; FERRARI, 2012), há três pressupostos teóricos:

1) Alguns estudos tentaram identificar o peso do ambiente na didática: em relaçâo à qualidade de um processo de aprendizagem, Hayward (1995) estimou que $75 \%$ encontra-se na predisposição do sujeito que aprende (motivação, memória) e $25 \%$ nos efeitos do "ambiente físico no qual se aprende";

2) A estrutura física e ambiente podem comunicar valores e desempenhar um papel na motivação (PROSHANSKY; WOLFE, 1974);

3) Os efeitos da educação estão relacionados à compatibilidade entre o estilo de ensino adotado e o ambiente físico (WEINSTEIN, 1981).

A integração desses pressupostos com os dados derivados de uma série de análises de experiências permitiu construir uma categorização dos ambientes das salas de aula digitais, com base da distribuição de tecnologias aos estudantes e na tipologia dos dispositivos utilizados. A categorização prevê sete tipologias de configuração de ambientes (GARAVAGLIA; FERRARI, 2012):

a. One-to-one/Um a um - ambiente tradicional em que a mesma tecnologia é distribuita a todos os alunos, no modelo um computador/artefato por aluno;

b. Pares frontais - ambiente tradicional em que a tecnologia é compartilhada por pares e a didática é voltada ao trabalho em pares;

c. Ilhas para grupos de trabalho - ambiente colaborativo baseado em ilhas de aprendizagem feitas com bancadas, com a tecnologia no meio e o professor que se movimenta supervisionando o trabalho;

d. Multitela - revisitação do ambiente precedente, em que o trabalho ocorre por tarefas paralelas e se utiliza as telas para organizar momentos de seminários;

e. Ilhas disciplinares - penínsulas para trabalhos disciplinares específicos realizados alternando aulas tradicionais com outras possibilidades em que o professor supervisiona o trabalho dos estudantes. Os bancos são organizados de ilhas para as ilhas, cada uma com materiais específicos de cada disciplina. As entregas podem ser alternadas com base na disponibilidade das ilhas;

f. Ilhas midiáticas - trata-se de bancadas organizadas conforme as características das tecnologias (por exemplo, as dedicadas à edição de vídeo com computadores e equipamentos específicos). $\mathrm{O}$ professor alterna aulas expositivas com trabalhos em 
grupo. As tarefas podem ser alternadas entre os grupos específicos, conforme a disponibilidade das ilhas;

g. Dispositivos móveis - é o ambiente mais interessante e inovador, que se obtém predispondo atividades externas que prevejam o uso de dispositivos móveis (tablets, smartphone). A supervisão do professor é realizada remotamente. É possível desenvolver didáticas "de campo" e os processos recuperam muitas das características das atividades informais usadas pelos estudantes com os mesmos dispositivos em seu tempo livre.

Tabela 1 - Categorizaçáo da configuraçáo do ambiente das salas de aula digitais

\begin{tabular}{|c|c|c|c|c|}
\hline Modelo & Cenário/ambiente & Proxemica & Tecnologia & Didática \\
\hline a- One-to-one & Tradicional & Tradicional & $\begin{array}{c}\text { Igual para todos, } \\
\text { distribuída }\end{array}$ & Tradicional \\
\hline $\begin{array}{l}\text { b. Ilhas de pares } \\
\text { frontais }\end{array}$ & Tradicional & Tradicional & $\begin{array}{c}\text { Compartilhada e } \\
\text { distribuída }\end{array}$ & $\begin{array}{l}\text { Trabalho em } \\
\text { pares }\end{array}$ \\
\hline $\begin{array}{l}\text { c. Ilhas para o } \\
\text { trabalho em equipe }\end{array}$ & $\begin{array}{c}\text { Ilhas de } \\
\text { aprendizagem }\end{array}$ & $\begin{array}{l}\text { Professor em movimento } \\
\text { para a supervisáo }\end{array}$ & $\begin{array}{l}\text { No centro do } \\
\text { grupo }\end{array}$ & Grupos \\
\hline d. Multitela & $\begin{array}{c}\text { Variante das } \\
\text { anteriores a, b, c } \\
\text { com telas maiores }\end{array}$ & $\begin{array}{l}\text { Com base na variação dos } \\
\text { ambientes anteriores }\end{array}$ & $\begin{array}{c}\text { a/b/c + telóes } \\
\text { distribuídos sobe } \\
\text { as }\end{array}$ & $\begin{array}{c}\text { Para tarefas } \\
\text { paralelas, se } \\
\text { prefere as } \\
\text { restituiçóes } \\
\text { com seminários }\end{array}$ \\
\hline $\begin{array}{l}\text { e. Ilhas } \\
\text { disciplinares }\end{array}$ & $\begin{array}{c}\text { Penínsulas para } \\
\text { trabalhos } \\
\text { disciplinares } \\
\text { específicos }\end{array}$ & $\begin{array}{c}\text { Alternância tradicional e } \\
\text { supervisão }\end{array}$ & $\begin{array}{l}\text { Dedicado por } \\
\text { disciplina }\end{array}$ & $\begin{array}{l}\text { Alternância das } \\
\text { tarefas } \\
\text { conforme a } \\
\text { disponibilidade }\end{array}$ \\
\hline f. Ilhas midiáticas & $\begin{array}{l}\text { Penínsulas para } \\
\text { trabalhar com } \\
\text { tecnologias } \\
\text { específicas }\end{array}$ & $\begin{array}{c}\text { Alternância tradicional e } \\
\text { supervisão }\end{array}$ & $\begin{array}{l}\text { Conforme as } \\
\text { potencialidades e } \\
\text { características }\end{array}$ & $\begin{array}{c}\text { Alternância das } \\
\text { tarefas } \\
\text { conforme a } \\
\text { disponibilidade } \\
\text { das ilhas }\end{array}$ \\
\hline $\begin{array}{l}\text { g. Dispositivos } \\
\text { Móveis }\end{array}$ & Fora da sala de aula & Supervisão remota & Móvel & $\begin{array}{l}\text { Não-formal / } \\
\text { no campo }\end{array}$ \\
\hline
\end{tabular}

Fonte: Síntese a partir de Garavaglia e Ferrari, 2012.

\section{Questões relacionadas ao design: uma análise insuficiente das necessidades}

As classificaçóes e as tendências aqui relatadas e descritas não são suficientes para entender o cenário ligado ao debate atual sobre o tema da renovaçáo da configuraçáo 
dos ambientes e dos modelos didáticos da escola com a introdução de novas mídias. Há outros fatores que estâo incidindo nas escolhas de planejamento, como, por exemplo, a limitada disponibilidade de orçamento por parte das escolas e uma ideia difusa e substancialmente ingênua de que através da aquisição de um grande número de tecnologias seja possível aumentar significativamente a qualidade de ensino (CALVANI, 1999).

Em um estudo recente realizado na Itália (GARAVAGLIA; PETTI, 2013), evidencia-se uma criticidade relacionada ao reduzido ou inexistente processo de análise das necessidades nos planejamentos que as escolas realizaram nos últimos anos, em resposta aos editais de financiamento das salas de aula digitais6.

Para entender a questão, é necessário recuperar a ideia de problem solving, resoluçấo de problemas, no design: os problemas são definidos por um conjunto de necessidades que devem ser atendidas a fim de transformar o estado da situação corrente (indesejada ou problemática) em um dos estados desejados (MUNARI, 1981). O problema é que, se não for posto em prática um processo de análise cuidadosa $\mathrm{e}$ identificaçáo das necessidades, quando a escola tiver a possibilidade de responder a uma chamada ou edital para a aquisição de tecnologia educativa, há o risco de identificar o estado desejado com a própria aquisição de tecnologias. Por outro lado, entender e identificar as necessidades de forma eficaz é, por si só, um problema bastante complexo (ARIELLI, 2003).que pode ser traduzida como o modelo 1:1 ou um computador por aluno.

Figura 3 - O curto-circuito do planejamento sem possibilidade de experimentaçâo do modelo resolutivo: a passagem para a soluçáo é direta e todo o orçamento disponível é gasto em uma solução que foi preparada apenas a nível teórico

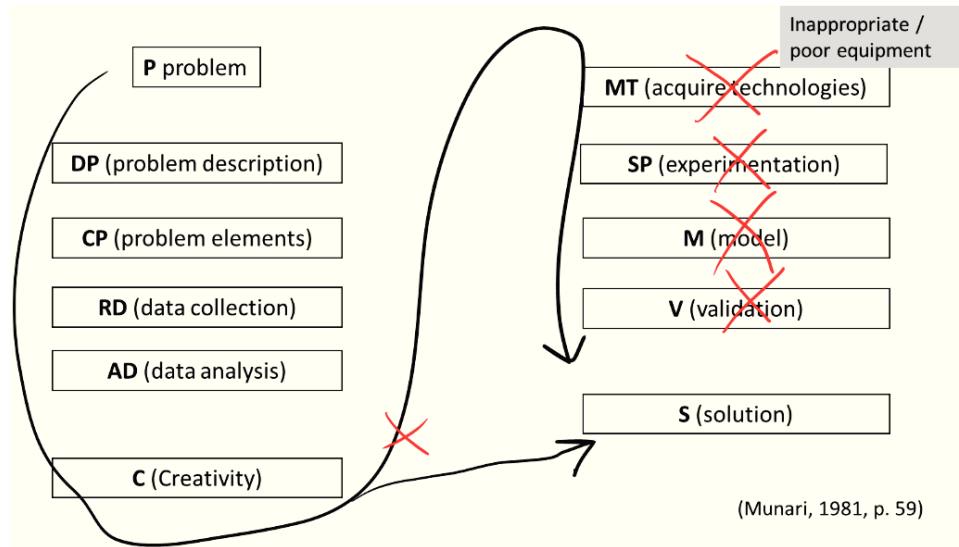

Fonte: Garavaglia e Petti, 2013, p. 256, com base em Munari, 1981, p. 59. 
Na Figura 3, evidencia-se uma síntese das análises realizadas sobre quatro dos mais importantes editais que promoveram o planejamento e a implementação das salas de aula digitais propostos pelo governo italiano de 2006 a 2013. A pesquisa foi realizada através da análise desses projetos que envolveram um total de 59 escolas e salas de aula digitais (31 nos anos iniciais, 20 nos anos finais do ensino fundamental, e oito no ensino médio) implementadas em escolas no norte da Itália.

A metodologia envolveu a análise de documentos, entrevistas com professores, grupos focais com alunos e questionários a professores e alunos. Nesses projetos, as chamadas dos editais não previram de modo explícito a possibilidade de experimentar, em um pequeno número, o modelo de planejamento proposto. E, em alguns casos, esta avaliação não era realmente viável, dadas as restrições impostas aos professores. $\mathrm{O}$ resultado é que a análise das necessidades, não sendo um pré-requisito específico e solicitado, tornou-se um processo que foi evitado, visto que o pedido se reduziu apenas às fases de uma proposta de projeto e a sua implementação. Assim, identifica-se um evidente curto-circuito no planejamento, pelo menos na fase de experimentaçáo do modelo pensado antes de adotá-lo em grande escala: isso significa efetivamente que as escolas tendem a construir o projeto e realizá-lo adquirindo rapidamente todas as tecnologias sem reservar recursos para experimentação e avaliação das soluçóes. Esta escolha implica que todos os recursos sejam assim gastos, com uma solução que não é realmente testada, aumentando o risco de adquirir tecnologias pouco úteis para as necessidades reais das turmas, das salas de aulas e das escolas. O risco pedagógico é, portanto, bastante elevado, visto que, desse modo, aumenta-se a probabilidade de construir projetos pouco significativos do ponto de vista educativo e didático.

\section{Conclusões}

Como poderia ser o próximo cenário? O que parece emergir no momento é uma notável disparidade de recursos entre as turmas de "série A", tecnologicamente equipadas, e as turmas de "séries inferiores", em que as tecnologias, no máximo, podem entrar se os alunos trouxerem e/ou levarem seus dispositivos. Este último caso, conhecido como BYOD apresenta algumas vantagens ligadas ao fato de que os alunos podem ser mais responsáveis, mas também interessados em conhecer melhor o dispositivo do qual dispóem. Em contrapartida, a soluçáo BYOD implica que o professor assuma a responsabilidade pela escolha de aplicativos compatíveis e multiplataformas, operação que requer competências técnicas mais elevadas para operar em segurança. Uma vez resolvido o problema da aquisição de recursos, ainda permanecem muitas situaçóes em que o planejamento não parece solidamente 
ancorado nas análises das necessidades, e, para tal, uma revisão dos processos de formação e atualização seria altamente desejável.

\section{Notas}

${ }^{1}$ Instituto Nacional de Documentação, Inovação e Pesquisa Educativa.

${ }^{2}$ N.T.: O termo Flipped Lesson tem sido utilizado no país com o sentido de "aulas invertidas", em que se inverte a sequência didática da aula expositiva feita pelo professor e depois a tarefa solicitada aos alunos, iniciando com as atividades solicitadas aos alunos para depois o professor fazer a síntese e/ou apresentação a partir do que os alunos fizeram. Esse conceito se aproxima da Lição a posteriori, que Celestin Freinet propôs ainda na década de 1940 .

${ }^{3}$ N.T.: A metodologia dos Episódios de Aprendizagem Situada (EAS) prevê a integração de dispositivos móveis na didática a partir do conceito de aprendizagens móveis e de microaprendizagens, em que a aula se organiza em três momentos: momento prévio e/ou preparatório, momento operativo e momento restruturador. Nessa proposta, os conteúdos são organizados em torno de atividades breves de produção dos alunos, com instruçóes prévias e reflexões posteriores feitas pelo professor, modificando o desenho da aula, o planejamento e a avaliação. Ver Rivoltella (2013).

${ }^{4}$ N.T.: Tais dados também se confirmam no contexto brasileiro. Ver resultados da pesquisa anual TIC Kids Online Brasil sobre o uso da Internet por crianças e adolescentes, realizada pelo Centro Regional de Estudos para o Desenvolvimento da Sociedade da Informação (Cetic.br), do Núcleo de Informação e Coordenação do Ponto BR (NIC.br).

${ }^{5}$ Ver: <http://vittra.se>.

${ }^{6}$ N.T.: Editais promovidos pelo governo italiano dirigidos às escolas, que participam com projetos especiais, a fim de equiparem suas salas de aula com tecnologias digitais e propostas de formação.

\section{REFERÊNCIAS}

ARIELLI, Emanuele. Pensiero e progettazione: la psicologia cognitiva applicata al design e all'architettura. Milano: Mondadori, 2003.

FULLER, Richard Buckminster. Nine chains to the moon. London: Cape, 1973.

BURKE, Karen; BURKE-SAMIDE, Barbara. Required changes in the classroom environment: it's a matter of design. The Clearing House, Winston-Salem, v. 77, n. 6, p. 236-239, 2004,

CALVANI, Antonio. I nuovi media nella scuola. Roma: Carocci, 1999. 
CROSS, Jay. Informal learning: rediscovering the natural pathways that inspire innovation and performance. San Francisco: Pfeiffer, 2006.

CUBAN, Larry. The open classroom. Education Next, Cambridge, v. 4, n. 2, 2004. Disponível em: <http://educationnext.org/theopenclassroom/>. Acesso em: $2 \mathrm{dez}$. 2014.

GARAVAGLIA, Andrea. Scuola senza classi? In: RIVOLTELLA, Pier Cesare. Fare didattica con gli EAS: episodi di Apprendimento Situato. Brescia : La Scuola, 2013. p. 37-41.

GARAVAGLIA, Andrea. Influenza dei processi transmediali nello sviluppo di LMS talent, mobile e social. In: GARAVAGLIA, Andrea. Transmedia Education: contenuti, significati, valori. Milano : Unicopli, 2014. p. 231-242.

GARAVAGLIA, Andrea; FERRARI, Simona. A model for defining digital classroom settings. Procedia: social \& behavioral sciences, Atlanta, v. 46, p. 1983-1987, 2012 GARAVAGLIA, Andrea; PETTI, Livia. Needs analysis in classroom digitalization projects. In: WINTER CONFERENCE LEARNING \& TEACHING WITH MEDIA \& TECHNOLOGY. 2013. Genoa. Proceedings... Genoa: ATEE, 2013. p. 251-258.

HYMAN, Adam. Managing the Digital Classroom: dozens of awesome teacher-tested ideas that help you manage and make the most of every digital tool in your classroom. New York: Scholastic Teaching Resources, 2014.

HAYWARD, Pamela. When novelty isn't enough: A case study of students' reactions to technology in the classroom environment. College Student Journal, Texas, v. 28, n. 3, p. 320-325, 1995.

JENKINS, Henry. Transmedia Education: the 7 Principles Revisited. Confessions of an Aca-Fan: the official weblog of Henry Jenkins, 21 jun. 2010. Disponível em: $<$ http://henryjenkins.org/2010/06/transmedia_education_the_7_pri.html>. Acesso em: 20 dez. 2014.

JOHN, Peter; WHEELER, Steve. The Digital Classroom: harnessing technology for the future of learning and teaching. London: Routledge, 2008.

KRAMER, Sibylle. Schools: Educational Spaces. Salenstein: Braun, 2009.

KUKULSKA-HULME Agnes; TRAXLER John. Design principle for mobile learning. In: BEETHAM, Helen; SHARPE, Rhona (Eds.). Rethinking pedagogy for a digital age: designing for 21 st century learning. 2. ed. London: Routledge, 2013. p. 244-257. 
MALCOM, Roberts. The digital classroom: the critical success factors. Saarbrucken: Lambert Academic Publishing, 2010.

MAZUR, Eric. Can we teach computers to teach? Computers in Physics, Melville, v. 5, p. 31-38, 1991.

MUNARI, Bruno. Da cosa nasce cosa. Bari: Laterza, 1981.

PENUEL, William R. Implementation and effects of one-to-one computing initiatives: a research synthesis. Journal of Research on Technology in Education, Eugene, v. 38, 329-348, 2006. Disponível em:

<https://www.chatsworth.com.sg/uploaded/PDF_Forms/PDF_Images/implementPBL.pdf >. Acesso em: 2 dez. 2014.

PONTEFRACT, Dan. Flat Army: creating a connected and engaged organization. Danvers: John Wiley \& Sons Inc, 2013.

PROSHANSKY, Etta; WOLFE, Maxine. The Physical Setting and Open Education. The School Review, Chicago, v. 82, n. 4, p.556-574, 1974. Disponível em: <http://www.jstor.org/stable/1084003>. Acesso em: 2 dez. 2014.

RIVOLTELLA, Pier Cesare. Fare didattica con gli EAS: episodi di Apprendimento Situato. Roma: Carocci, 2013.

RIVOLTELLA, Pier Cesare et al. Could technology encourage innovation in school? An overview of "Classi 2.0" project in Lombardia (Italy). Research on Education and Media, Berlin, v. 4, n. 2, p. 253-264, 2012.

SILBERMAN, Charles. The open classroom reader. New York: Random House, 1973.

THE NIELSEN COMPANY. The digital consumer February 2014. New York: Nielsen, 2014. Disponível em: <http://www.nielsen.com/content/dam/corporate/us/en/reportsdownloads/2014\%20Reports/the-digital-consumer-report-feb-2014.pdf >. Acesso em: 20 dez. 2014.

WEINSTEIN, Carol. Classroom design as an external condition for learning. Educational Technology, v. 21, n. 8, p.12-19, 1981. 


\section{Mobile device at school: rethinking environments and methodologies in the Italian context}

\begin{abstract}
This article discusses some recent studies that allow us to understand the importance of analyzing the educational needs in the conceiving of the digital classroom and its possible redefinitions in the learning and methods environment. This paper also proposes a reflection on the implementation of innovative educational environments and settings through mobile technologies in Italian schools. Recently, the focus on active teaching and informal learning with the integration of mobile devices has reflected in the current Flipped Lesson and in the Episodes of Situated Learning EAL, some of the most interesting methods to support the renovation of school practice. These methods are based on group work both in the classroom and at home with digital devices and BYOD Bring Your Own Device model. Choosing the right teaching method to the current challenges is fundamental in a society where the use of the internet increases every day and the possibility of being always connected provides diffuse learning processes.
\end{abstract}

Keywords: School. Device mobile. Learning.
Mobile device a scuola: ripensare gli ambienti e i metodi per l'apprendimento nel contesto italiano

\section{Riassunto}

Recenti studi hanno messo in evidenza l'importanza dell'analisi dei bisogni nella progettazione delle classi digitali. Questo articolo propone una riflessione sulla realizzazione di ambienti didattici innovativi in relazione agli impianti didattici che tengono conto dell'incremento della diffusione dei device mobile nella scuola italiana. Recentemente l'attenzione sulle didattiche attive e sugli apprendimenti informali integrati all'uso dei device mobile ha avuto particolare riscontro nelle correnti della Flipped lesson e degli Episodi di Apprendimento Situato, EAS, che si stanno posizionando come alcuni dei metodi più interessanti per sostenere il rinnovamento delle pratiche scolastiche. Questi metodi sono basati sul lavoro di gruppo sia in aula che a casa con i dispositivi digitali e sul modello BYOD Bring Your Own Device (porta il tuo device a scuola). La scelta del metodo di insegnamento è perciò fondamentale in una società in cui l'uso di internet è in crescita e la possibilità di essere sempre più collegati durante l'intera giornata permettono processi di Pervasive Learning.

Parole chiave: Scuola. Dispositivi mobili. Apprendimento. 


\section{Andrea Garavaglia}

E-mail: andrea.garavaglia@unimib.it

\section{Monica Fantin}

E-mail: fantin.monica@gmail.com

Recebido em: 05/02/2015

Aprovado em: 13/03/2015 